\title{
Solutions of the Time-Dependent Klein-Gordon and Dirac Equations for a Uniform Electric Field
}

\author{
Vernon W. Myers \\ Institute for Materials Research, National Bureau of Standards, Washington, D.C. 20234
}

(October 12, 1967)

\begin{abstract}
The time-dependent Klein-Gordon and Dirac equations are solved for the motion of a charged particle in a classical uniform electrostatic field of infinite extent. The elementary solutions have a position dependence of the form $e^{i \mathbf{k} \cdot \mathbf{r}}$ with the component of $k$ in the field direction varying linearly with time.
\end{abstract}

Key Words: Dirac and Klein-Gordon equations, time-dependent solutions, uniform electric field.

\section{Introduction}

Electromagnetic forces have the proper behavior under a Lorentz transformation, and this paper treats the relativistic equations for the motion of a charged particle in a uniform electrostatic field of infinite extent. The interaction of a charged particle with a quantized field is of great interest, but any thorough treatment is rather involved and necessarily requires approximations. The field will be treated classically, and such effects as polarization of the vacuum and radiation by the accelerated charge are not included. This example of a uniform electric field is chosen, because the time-dependent equations describing traveling waves can be solved in a rather straightforward manner.

It is worthwhile to examine the classical equations of motion before undertaking the quantum mechanical treatment. The uniform electrostatic field $E$ is taken to be in the positive $x$ direction. The gage may be chosen arbitrarily, and it does not affect any of the observables. Maxwell's equation relating $E$ to the scalar and vector potentials is

$$
\mathbf{E}=-\nabla V-\frac{1}{c} \frac{\partial \mathbf{A}}{\partial t}
$$

An obvious gage is $V=-E x, A=0$ for an electrostatic field $\mathbf{E}=\hat{i} E$ ( $E$ a constant). If this gage is used, the relativistic Hamiltonian for a particle of rest mass $m$ and charge $\epsilon$ is

$$
H=\left[m^{2} c^{4}+c^{2}\left(p_{x}^{2}+p_{y}^{2}+p_{z}^{2}\right)\right]^{1 / 2}-\epsilon E x .
$$

Hamilton's equations are

$$
\dot{p}_{x}=-\frac{\partial H}{\partial x}, \dot{x}=\frac{\partial H}{\partial p_{x}}, \text { etc. }
$$

One finds that the various components of momentum are

$$
p_{x}=p_{0 x}+\epsilon E t, p_{y}=p_{0 y}, p_{z}=p_{0 z}
$$


where $p_{0 x}, p_{0 y}, p_{0 z}$ are constants. The linear time dependence for $p_{x}$ is the same as the nonrelativistic result and is due to the fact that the electric field is uniform. The traveling wave solutions of the quantum mechanical equation have the same time dependence for the momentum as the classical relations, of course.

An alternative gage is $V=0, \mathbf{A}=-\hat{i} c E t$. The $x$-component of mechanical momentum would be

$$
\frac{m v_{x}}{\left(1-\frac{v^{2}}{c^{2}}\right)^{1 / 2}}=p_{x}-\frac{\epsilon}{c} A_{x}=p_{x}+\epsilon E t .
$$

The Hamiltonian would be

$$
H=\left[m^{2} c^{4}+c^{2}\left[\left(p_{x}+\epsilon E t\right)^{2}+p_{y}^{2}+p_{z}^{2}\right]\right]^{1 / 2}
$$

where $p_{x}, p_{y}, p_{z}$ are constants of motion. The original gage $V=-E x, \mathbf{A}=0$ is preferable because $p$ is then the mechanical momentum. In addition, the introduction of the vector potential does not simplity the solution of the quantum mechanical equations.

\section{Klein-Gordon Equation}

The scalar wave equation is appropriate for a spin zero particle, a charged $\pi$ meson, for example. The wave equation is found by taking the classical relation

$$
(H+\epsilon E x)^{2}=m^{2} c^{4}+c^{2} p^{2},
$$

and applying it in operator form. The Klein-Gordon equation is

$$
\left(i \hbar \frac{\partial}{\partial t}+\epsilon E x\right)^{2} \psi=\left(m^{2} c^{4}-c^{2} \hbar^{2} \nabla^{2}\right) \psi
$$

Let

$$
\psi=e^{i \mathbf{k} \cdot \mathbf{r}} T
$$

where $k_{x}$ and $T$ are functions of the time. The quantum mechanical expression for momentum is identical with the classical results, eq (3). That is,

$$
k_{x}=k_{0 x}+\frac{\epsilon E t}{\hbar}, k_{y}=k_{0 y}, k_{z}=k_{0 z}
$$

where $k_{0 x}, k_{0 y}, k_{0 z}$ are constants. The linear time dependence of $k_{x}$ is required if $T$ is to be a function of the time only. The function $e^{i \mathbf{k} \cdot \mathbf{r}}$ represents a state in which the momentum and kinetic energy are known with complete certainty since it is an eigenfunction for both the momentum and kinetic energy operators.

The equation for $T$ is found from the substitution of eq (8) into eq (7):

$$
-\hbar^{2} \frac{d^{2} T}{d t^{2}}=\left[m^{2} c^{4}+c^{2} \hbar^{2}\left(k_{x}^{2}+k_{y}^{2}+k_{z}^{2}\right)\right] T
$$

In the limit of $E \rightarrow 0, T$ is $e^{ \pm \frac{i W t}{\hbar}}$ where $W$ is the total energy. It is possible to find a power series solution for $T$. If the dimensionless quantities 


$$
\begin{aligned}
u & =\left(\frac{c \epsilon E}{\hbar}\right)^{1 / 2}\left(t+\frac{k_{0 x} \hbar}{\epsilon E}\right)=\left(\frac{c \hbar}{\epsilon E}\right)^{1 / 2} k_{x}, \\
a^{2} & =\frac{m^{2} c^{3}+c \hbar^{2}\left(\dot{k}_{y}^{2}+k_{z}^{2}\right)}{\epsilon E \hbar}
\end{aligned}
$$

are used, then

$$
\frac{d^{2} T}{d u^{2}}+\left(u^{2}+a^{2}\right) T=0
$$

It is interesting to note that eq (12) is the same as the nonrelativistic Schrödinger equation for the potential $-u^{2}$. The solution of (12) can be expressed in the following way:

$$
T=\exp \left( \pm \frac{i u^{2}}{2}\right) \sum_{0}^{\infty} b_{n} u^{n}
$$

The coefficients of the series are determined by the recursion relation

$$
\frac{b_{n+2}}{b_{n}}=\mp \frac{i(2 n+1)+a^{2}}{(n+1)(n+2)}
$$

for both odd and even $n$. The series converges for all finite values of $t$. The coefficients $b_{0}$ and $b_{1}$ are arbitrary. The signs in the foregoing equations are associated with positive and negative charges [1]. That is, if $e^{\frac{i u^{2}}{2}}$ corresponds to a positively charged meson with positive kinetic energy, then $e^{-\frac{i u^{2}}{2}}$ corresponds to a negatively charged meson with positive kinetic energy.

Equation (10) is very similar to the time-independent relation

$$
(W+\epsilon E x)^{2} \psi=\left(m^{2} c^{4}-c^{2} \hbar^{2} \nabla^{2}\right) \psi
$$

Let $\psi=e^{i\left(k_{y} y+k_{z} z\right)} \phi(x)$.

Then,

$$
(W+\epsilon E x)^{2} \phi=\left[m^{2} c^{4}+c^{2} \hbar^{2}\left(k_{y}^{2}+k_{z}^{2}\right)\right] \phi-c^{2} \hbar^{2} \frac{d^{2} \phi}{d x^{2}} .
$$

Time and distance enter in a symmetrical way for this example of a uniform field.

\section{Dirac Equation}

The Dirac equation is for a spin $1 / 2$ particle, an electron for instance. The time-dependent equation is

$$
H \psi=i \hbar \frac{\partial \psi}{\partial t}
$$

The Dirac Hamiltonian is

$$
H=-i \hbar c\left(\alpha_{x} \frac{\partial}{\partial x}+\alpha_{y} \frac{\partial}{\partial y}+\alpha_{z} \frac{\partial}{\partial z}\right)+\beta m c^{2}-\epsilon E x .
$$


The common representation is used:

$$
\begin{aligned}
\alpha_{x}=\left(\begin{array}{cccc}
0 & 0 & 0 & 1 \\
0 & 0 & 1 & 0 \\
0 & 1 & 0 & 0 \\
1 & 0 & 0 & 0
\end{array}\right), & \alpha_{y}=\left(\begin{array}{cccc}
0 & 0 & 0 & -i \\
0 & 0 & i & 0 \\
0 & -i & 0 & 0 \\
i & 0 & 0 & 0
\end{array}\right), \\
\alpha_{z}=\left(\begin{array}{cccc}
0 & 0 & 1 & 0 \\
0 & 0 & 0 & -1 \\
1 & 0 & 0 & 0 \\
0 & -1 & 0 & 0
\end{array}\right), & \beta=\left(\begin{array}{cccc}
1 & 0 & 0 & 0 \\
0 & 1 & 0 & 0 \\
0 & 0 & -1 & 0 \\
0 & 0 & 0 & -1
\end{array}\right) .
\end{aligned}
$$

Let us assume the solution

$$
\psi=\left(\begin{array}{c}
A_{1} \\
A_{2} \\
A_{3} \\
A_{4}
\end{array}\right) e^{i \mathbf{k} \cdot \mathbf{r}}
$$

where the $A_{\lambda}$ are functions of the time and $k_{x}=k_{0 x}+\frac{\epsilon E t}{\hbar}$ as before. For convenience, $k_{z}$ will be taken to be zero to simplify the spinor equations. This does not cause any loss of generality. The substitution of eq (20) into (19) yields

$$
\hbar c k_{x}\left(\begin{array}{c}
A_{4} \\
A_{3} \\
A_{2} \\
A_{1}
\end{array}\right)+i \hbar c k_{y}\left(\begin{array}{c}
-A_{4} \\
A_{3} \\
-A_{2} \\
A_{1}
\end{array}\right)+m c^{2}\left(\begin{array}{c}
A_{1} \\
A_{2} \\
-A_{3} \\
-A_{4}
\end{array}\right)=-i \hbar \frac{d}{d t}\left(\begin{array}{c}
A_{1} \\
A_{2} \\
A_{3} \\
A_{4}
\end{array}\right) .
$$

This is equivalent to the relations

$$
\begin{aligned}
& -i \frac{d A_{1}}{d t}+\frac{m c^{2}}{\hbar} A_{1}+c\left(k_{x}-i k_{y}\right) A_{4}=0, \\
& -i \frac{d A_{2}}{d t}+\frac{m c^{2}}{\hbar} A_{2}+c\left(k_{x}+i k_{y}\right) A_{3}=0, \\
& -i \frac{d A_{3}}{d t}-\frac{m c^{2}}{\hbar} A_{3}+c\left(k_{x}-i k_{y}\right) A_{2}=0, \\
& -i \frac{d A_{4}}{d t}-\frac{m c^{2}}{\hbar} A_{4}+c\left(k_{x}+i k_{y}\right) A_{1}=0 .
\end{aligned}
$$


It is seen that $A_{1}$ and $A_{4}$ are coupled and $A_{2}$ and $A_{3}$ are coupled.

These equations can be made more symmetrical by taking the linear combinations

$$
\phi_{1}=A_{1}+A_{4}, \quad \phi_{2}=A_{2}+A_{3}, \quad \phi_{4}=A_{1}-A_{4}, \quad \phi_{3}=A_{2}-A_{3} .
$$

Then we obtain

$$
\begin{aligned}
& -i \frac{d \phi_{1}}{d t}+c k_{x} \phi_{1}+\left(\frac{m c^{2}}{\hbar}+i c k_{y}\right) \phi_{4}=0, \\
& -i \frac{d \phi_{4}}{d t}-c k_{x} \phi_{4}+\left(\frac{m c^{2}}{\hbar}-i c k_{y}\right) \phi_{1}=0, \\
& -i \frac{d \phi_{2}}{d t}+c k_{x} \phi_{2}+\left(\frac{m c^{2}}{\hbar}-i c k_{y}\right) \phi_{3}=0, \\
& -i \frac{d \phi_{3}}{d t}-c k_{x} \phi_{3}+\left(\frac{m c^{2}}{\hbar}+i c k_{y}\right) \phi_{2}=0 .
\end{aligned}
$$

These spinor components have a somewhat similar time-dependence as the Klein-Gordon case. This is illustrated by examining $\phi_{1}$ and $\phi_{4}$. If the dimensionless quantities, eq (11), are used and the substitutions

$$
\phi_{1}=e^{\frac{i u^{2}}{2}} f, \quad \phi_{4}=e^{\frac{i u^{2}}{2}} g
$$

are made, then

$$
-i \frac{d f}{d u}+2 u f+\left(\frac{\hbar}{c \epsilon E}\right)^{1 / 2}\left(\frac{m c^{2}}{\hbar}+i c k_{y}\right) g=0, \quad-i \frac{d g}{d u}+\left(\frac{\hbar}{c \epsilon E}\right)^{1 / 2}\left(\frac{m c^{2}}{\hbar}-i c k_{y}\right) f=0 .
$$

These equations are equivalent to

$$
\frac{d^{2} f}{d u^{2}}+2 i \frac{d}{d u}(u f)+a^{2} f=0, \quad g=-i\left(\frac{\hbar}{c \epsilon E}\right)^{1 / 2}\left(\frac{m c^{2}}{\hbar}-i c k_{y}\right) \int f d u+g_{0} .
$$

The function $f$ can be represented by a power series,

$$
f=\sum_{0}^{\infty} b_{n} u^{n}
$$

The coefficients of the series are found from the recursion relation

$$
\frac{b_{n+2}}{b_{n}}=-\frac{2 i(n+1)+a^{2}}{(n+1)(n+2)}
$$

for both odd and even $n$. The two constants $b_{0}$ and $b_{1}$ are determined by the initial values of $f$ and $g$ at zero time. The functions $f$ and $g$ are interchanged in eq (26) and all terms containing $i$ change sign if $e^{-\frac{i u^{2}}{2}}$ is used (charge conjugation). The components $A_{1}$ and $A_{4}$ are given by

$$
\begin{aligned}
& A_{1}=\frac{1}{2} e^{ \pm \frac{i u^{2}}{2}}(f+g), \\
& A_{4}=\frac{1}{2} e^{ \pm \frac{i u^{2}}{2}}(f-g) .
\end{aligned}
$$


The components $A_{2}$ and $A_{3}$ can be expressed in a similar way. The solution of the time independent Dirac equation for a uniform field has been treated by Sauter [2] who has a detailed description of the solution of equations similar in form to eq (26).

The two arbitrary constants $b_{0}$ and $b_{1}$ for the Klein-Gordon solution and $f_{0}$ and $g_{0}$ for the Dirac solution can be specified by normalizing the probability per unit volume and by the relative amounts of positive and negative charge at the initial time. For example, it would be possible to start with a "pure" charge at zero time. The charge could be positive (with no negative charge density) or negative (with no positive charge density). At later time, both charge densities are present, and the charge densities vary with time, but the net charge density is constant,

$$
\rho=\rho_{+}-\rho_{-}=\text {constant }
$$

These charge states are discussed by Feshbach [1] and by Foldy [3].

This examination of the time-dependent equations could be extended to the example of combined electric and magnetic fields. There are two Lorentz invariants [4],

$$
\begin{aligned}
& E^{2}-B^{2}=I_{1}, \\
& \mathbf{E} \cdot \mathbf{B}=I_{2} .
\end{aligned}
$$

If $\mathbf{E} \cdot \mathbf{B}=0$, but $I_{1} \neq 0$, it is possible to find a Lorentz frame in which there is only an electric or a magnetic field. If $E>B$ (Gaussian units), a frame exists in which there is only an electric field. If $E<B$, a frame exists in which there is only a magnetic field.

\section{References}

[1] H. Feshbach and F. Villars, Revs. Modern Phys. 30, 24 (1958).

[2] F. Sauter, Z. Physik 69, 742 (1931).

[3] Quantum Theory, III, 40, Ed. by D. R. Bates (Academic Press, New York, N.Y., 1962).

[4] W. Panofsky and M. Phillips, Classical Electricity and Magnetism, p. 362 (Addison-Wesley Publishing Company, Reading, Mass., 1955).

(Paper 72B1-258) 